\title{
Effects of Telmisartan on Arterial Stiffness Assessed by the Cardio-Ankle Vascular Index in Hypertensive Patients
}

\author{
Kenichiro Kinouchi ${ }^{a}$ Atsuhiro Ichiharab Mariyo Sakoda ${ }^{a} \quad$ Asako Kurauchi-Mito $^{a}$ \\ Kanako Murohashi-Bokuda ${ }^{a}$ Hiroshi Itoha \\ Departments of andocrinology, Metabolism, and Nephrology, and ${ }^{b}$ Endocrinology and Anti-Aging Medicine and \\ Internal Medicine, Keio University School of Medicine, Tokyo, Japan
}

\section{Key Words}

Albuminuria · Telmisartan · Arterial stiffness $\cdot$ Blood pressure $\cdot$ Cardio-ankle vascular index

\begin{abstract}
Background/Aims: This study was conducted to determine the effect of telmisartan on the cardio-ankle vascular index (CAVI), a novel blood pressure (BP)-independent marker for arterial stiffness in hypertensive patients. Methods: One hundred consecutive hypertensive patients were randomly assigned either to a group treated with calcium channel blocker (CCB)-based therapy or a group treated with telmisartan-based therapy. Clinical and biological parameters were then measured before and 12 months after the start of this study. Results: CAVI, the logarithm of urinary albumin excretion, and BP were reduced significantly after telmisartan-based therapy. The decreases in 24-hour diastolic BP and daytime systolic BP associated with telmisartan-based therapy were significantly greater than those associated with CCB-based therapy. Both therapies significantly and similarly decreased the clinical BP, 24-hour systolic BP, daytime diastolic BP and serum levels of low-density lipoprotein cholesterol. No significant differences in the metabolic parameters were observed between the two therapies. Conclusion:
\end{abstract}

Telmisartan-based therapy had beneficial effects on arterial stiffness assessed by CAVI, albuminuria, 24-hour BP and metabolism compared with CCB-based therapy. Since these markers are known to influence the future risk of cardiovascular events, telmisartan could be a useful drug for hypertensive patients.

Copyright $\odot 2010$ S. Karger AG, Basel

\section{Introduction}

Arterial stiffness is a powerful independent marker of vascular target organ damage and an independent prognostic predictor for cardiovascular morbidity and mortality [1]. In addition, arterial stiffness has been implicated as a marker for vasculature aging [2]. Its prevention and control are important in light of the growing number of patients with diabetes and hypertension. Arterial stiffness has been assessed using several methods, including pulse pressure, the augmentation index (AI) and pulse wave velocity (PWV). These parameters can easily be measured in the clinic in a matter of minutes using relatively inexpensive technology and a simple reproducible method. Each of these simple measures of arterial aging is correlated with the risk of cardiac, cerebral and vascu-

\section{KARGER}

Fax +4161306 1234 E-Mail karger@karger.ch www.karger.com

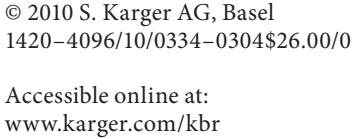

Atsuhiro Ichihara, $\mathrm{MD}, \mathrm{PhD}, \mathrm{FAHA}$

Endocrinology and Anti-Aging Medicine, Keio University School of Medicine

35 Shinanomachi, Shinjuku-ku

Tokyo 160-8582 (Japan)

Tel. +81 35363 3796, Fax +81 33359 2745, E-Mail atzichi@sc.itc.keio.ac.jp 
lar events $[1,3,4]$. However, these measures cannot be used to determine whether the improvement in arterial stiffness is caused by a blood pressure (BP)-dependent effect or a BP-independent mechanism. The cardio-ankle vascular index (CAVI) is a new marker of arterial stiffness that is calculated from the PWV and adjusted according to the BP value [5]. CAVI has been shown to be closely correlated with other markers contributing to arterial stiffness, such as the carotid intima-media thickness (IMT) [6].

Several lines of experimental and clinical evidence have documented that the renin-angiotensin system (RAS) plays a key role in the pathophysiology of a number of cardiovascular diseases. Angiotensin II type 1 receptor blocker (ARB) is currently one of the most wildly used first-line antihypertensive drugs. Evidence shows that it not only reduces BP, but also has some ancillary effects on cardiovascular properties, such as the protection of heart and kidney function beyond the mere lowering of elevated BP [7]. In particular, ARB telmisartan is reported to have an agonistic activity on peroxisome proliferator-activated receptor gamma (PPAR- $\gamma$ ), thereby ameliorating progression or even causing the regression of arterial stiffness [8]. In fact, treatment with telmisartan (40 $\mathrm{mg} /$ day for 3 months) in hypertensive patients decreased both BP and PWV [9]. However, whether telmisartan improves CAVI, which is less dependent on BP [5], remains uncertain.

The aim of this study was to assess the effects of an ARB telmisartan on markers of arterial stiffness using CAVI, AI and the maximum of the carotid intima-media thickness (MAX-IMT), along with urinary albumin excretion (UAE) and brain natriuretic peptide (BNP), which are known surrogate markers for cardiovascular morbidity and mortality in hypertensive patients [10].

\section{Patients and Methods}

\section{Study Population and Design}

The subjects of the present study were comprised of 100 consecutive patients with untreated hypertension or uncontrollable hypertension treated with medications other than RAS inhibitors. Hypertension was defined as follows. In patients without comorbid illness, hypertension was defined as a clinical systolic BP of $>140 \mathrm{~mm} \mathrm{Hg}$ and/or a clinical diastolic BP of $>90 \mathrm{~mm} \mathrm{Hg}$ at any time, and/or a systolic ambulatory $\mathrm{BP}$ of $>130 \mathrm{~mm} \mathrm{Hg}$ and/or a diastolic ambulatory BP of $>85 \mathrm{~mm} \mathrm{Hg}$ in the morning. In patients with diabetes mellitus, hypertension was defined as a clinical systolic BP of $>130 \mathrm{~mm} \mathrm{Hg}$ and/or a clinical diastolic BP of $>80 \mathrm{~mm} \mathrm{Hg}$ at any time.

Effects of Telmisartan on Arterial Stiffness
All the patients were randomly assigned to either a group treated with calcium channel blocker (CCB)-based therapy or a group treated with telmisartan-based therapy. The randomization was performed by the envelop method. The target BP was defined as $<130 / 85 \mathrm{~mm} \mathrm{Hg}$ in patients without any complications and $<130 / 80 \mathrm{~mm} \mathrm{Hg}$ in patients with diabetes mellitus, chronic kidney disease or metabolic syndrome. Patients treated with CCB-based therapy were first treated with $20 \mathrm{mg} /$ day of nifedipine, $2.5 \mathrm{mg}$ /day of amlodipine, $5 \mathrm{mg}$ /day of cilnidipine or $2 \mathrm{mg} /$ day of benidipine for 4 weeks, and the doses of the CCBs were subsequently increased every 4 weeks until the target BP was attained (up to $60 \mathrm{mg} /$ day of nifedipine, $10 \mathrm{mg} /$ day of amlodipine, $20 \mathrm{mg}$ /day of cilnidipine and $8 \mathrm{mg} /$ day of benidipine). If the target $\mathrm{BP}$ was not achieved, ARBs, $\beta$-blockers, diuretics and ACE inhibitors were subsequently added. Patients treated with telmisartan-based therapy were first treated with $20 \mathrm{mg}$ /day of telmisar$\tan$ for 4 weeks, and the telmisartan dose was subsequently increased every 4 weeks until the target BP was attained (up to a maximum dose of $80 \mathrm{mg} /$ day). The CCBs and diuretics were subsequently added unless the BP fell below the target BP. Clinical and biological parameters were measured before and 12 months after the start of this study. During the study period, previous medications and therapies other than antihypertensive drugs were continued. The subjects were not blinded to the treatment, although all measurements were performed under blind conditions.

The study was approved by the review board of Keio University Medical School Hospital and written informed consent was obtained from every subject.

Serum levels of creatinine, potassium, uric acid, triglyceride, high-density lipoprotein cholesterol, low-density lipoprotein cholesterol (LDL-C), glucose and glycoalbumin, and plasma levels of BNP, the active renin concentrations (ARC) and aldosterone (PAC) were measured in venous blood samples. The logarithm of urinary albumin excretion (log UAE) was measured in urinary samples drawn on the morning after an overnight fast on the same days as the ankle-brachial index (ABI), CAVI, AI, BP and MAXIMT measurements were performed.

\section{Ambulatory BP Monitoring}

An oscillometric-based device (TM-2431; A\&D Co., Tokyo, Japan) was used to perform 24-hour ambulatory BP monitoring. BP was measured every $30 \mathrm{~min}$ during the day (between 6:00 a.m. and 10:00 p.m.) and every $60 \mathrm{~min}$ during the night (between 10:00 p.m. and 6:00 a.m.). The mean values and the standard deviations of the ambulatory BP for each subject were calculated for a 24-hour period. The standard deviation of the ambulatory BP values was recorded as the variability of ambulatory BP in this study. The nocturnal decrease in BP was calculated as the average systolic BP during the day minus the average systolic BP during the night. The morning BP surge was calculated as the highest systolic BP during the first 2 hours after waking minus the lowest systolic BP during the night.

\section{Cardio-Ankle Vascular Index}

CAVI was measured using a VaSera VS-1000 vascular screening system (Fukuda Denshi Co. Ltd., Tokyo, Japan), as described previously [11]. Cuffs were applied to bilateral upper arms and ankles, with the subjects lying in a supine position with their heads held along the midline. ECG electrodes were placed on both 
wrists and a microphone for detecting heart sounds was placed over the sternum. The patients rested in this supine position for at least $10 \mathrm{~min}$ before the start of monitoring. CAVI was calculated using the following formula:

$$
\mathrm{CAVI}=\mathrm{a}\left\{(2 \rho / \Delta \mathrm{P}) \times \ln (\mathrm{Ps} / \mathrm{Pd}) \mathrm{PWV}^{2}\right\}+\mathrm{b},
$$

where Ps is the systolic BP, Pd is the diastolic BP, $\Delta \mathrm{P}$ is $\mathrm{Ps}-\mathrm{Pd}, \rho$ is blood density, and a and $\mathrm{b}$ are constants.

\section{Augmentation Index}

AI was measured using an automated tonometric device (HEM-9000AI; Omron Healthcare Co. Ltd., Kyoto, Japan), as described previously [12]. Peripheral pressure waveforms were recorded over $30 \mathrm{~s}$ from the radial artery at the wrist with the subjects in a sitting position after resting for at least $5 \mathrm{~min}$. AI was calculated using the following formula:

$$
\begin{aligned}
& \mathrm{AI}=(\text { late systolic } \mathrm{BP}-\text { diastolic } \mathrm{BP}) /(\text { systolic } \mathrm{BP}-\text { diastolic } \mathrm{BP}) \\
& \times 100(\%) .
\end{aligned}
$$

\section{Urinary Albumin Excretion}

UAE was evaluated on the basis of the mean albumin-to-creatinine ratio in three nonconsecutive overnight urine samples. The urinary concentrations of albumin and creatinine were determined using a turbidimetric immunoassay with a SuperiorMicroalbumin kit (DPC Co., Tokyo, Japan) and with the Jaffé reaction using an autoanalyzer.

\section{Carotid Intima-Media Thickness}

Ultrasonography B-mode imaging of the carotid artery was performed using a PowerVision 6000 machine (Toshiba, Tokyo, Japan) at a transducer frequency of $7.5 \mathrm{MHz}$. Each subject was examined while in a supine position. Up to $4 \mathrm{~cm}$ of the common carotid artery and the carotid bulb were scanned bilaterally using longitudinal and transverse projections. The images were focused on the far wall of the artery. IMT was defined as the distance between the leading edge of the lumen-intima interface and the leading edge of the media-adventitia interface of the far wall, and the greatest IMT value in the bilateral longitudinal projections was recorded as the maximum IMT. The IMT was measured within plaque-free areas. The mean intraobserver and interobserver coefficients of variation for the maximum IMT were 4.3 and $4.7 \%$, respectively.

\section{Statistical Analyses}

Analyses were performed using Microsoft Office Excel 2007 and StatView 5.0. software (SAS Institute Inc., Cary, N.C., USA). Fisher's exact test was used to analyze sex and the frequency of diabetes mellitus, smoking and the use of statins. The MannWhitney $U$ test was used to analyze age and body mass index. Changes in biological parameters were analyzed using Student's $t$ test and a two-way analysis of variance for repeated measures combined with Tukey-Kramer post hoc tests. The contributions of changes in variables to changes in CAVI were tested using a regression analysis and an analysis of covariance. A p value $<0.05$ was considered significant. Data are presented as the means \pm

\begin{tabular}{|c|c|c|c|}
\hline Characteristics & CCB-based & $\begin{array}{l}\text { Telmisartan- } \\
\text { based }\end{array}$ & $\mathrm{p}$ \\
\hline Number & 50 & 50 & 0.999 \\
\hline Age, years & $51.0 \pm 10.0$ & $52.4 \pm 8.7$ & 0.615 \\
\hline Male gender, $\mathrm{n}$ & 35 & 39 & 0.495 \\
\hline BMI & $25.4 \pm 4.1$ & $24.1 \pm 3.7$ & 0.412 \\
\hline $\mathrm{WC}, \mathrm{cm}$ & $86.9 \pm 9.9$ & $89.0 \pm 8.1$ & 0.452 \\
\hline $\mathrm{DM}, \mathrm{n}$ & 6 & 7 & 0.999 \\
\hline Smoker, $\mathrm{n}$ & 14 & 14 & 0.999 \\
\hline Use of statins, $\mathrm{n}$ & 12 & 10 & 0.810 \\
\hline Serum Cr, mg/dl & $0.88 \pm 0.34$ & $0.84 \pm 0.14$ & 0.539 \\
\hline Serum K, mEq/l & $4.3 \pm 0.3$ & $4.4 \pm 0.4$ & 0.062 \\
\hline Serum UA, mg/dl & $6.2 \pm 1.4$ & $6.1 \pm 1.5$ & 0.626 \\
\hline Serum TG, mg/dl & $165 \pm 126$ & $166 \pm 128$ & 0.964 \\
\hline Serum HDL-C, mg/dl & $57 \pm 15$ & $53 \pm 13$ & 0.213 \\
\hline Serum LDL-C, mg/dl & $130 \pm 34$ & $121 \pm 24$ & 0.142 \\
\hline $\mathrm{GA}, \%$ & $14.5 \pm 1.9$ & $14.7 \pm 1.8$ & 0.609 \\
\hline $\mathrm{BNP}, \mathrm{pg} / \mathrm{ml}$ & $11.0 \pm 8.3$ & $11.2 \pm 11.9$ & 0.927 \\
\hline Plasma ARC, pg/ml & $12.1 \pm 17.0$ & $9.6 \pm 8.7$ & 0.363 \\
\hline $\log \mathrm{UAE}$ & $1.16 \pm 0.69$ & $1.03 \pm 0.66$ & 0.445 \\
\hline $\mathrm{ABI}$ & $1.13 \pm 0.13$ & $1.16 \pm 0.08$ & 0.188 \\
\hline CAVI & $7.8 \pm 1.3$ & $8.2 \pm 1.0$ & 0.051 \\
\hline $\mathrm{AI}, \%$ & $80.1 \pm 16.2$ & $83.7 \pm 13.6$ & 0.408 \\
\hline $\max$ IMT, mm & $1.1 \pm 0.8$ & $1.2 \pm 0.6$ & 0.394 \\
\hline
\end{tabular}
SD.
Table 1. Patient characteristics at baseline

Data are the means $\pm \mathrm{SD}$. WC $=$ Waist circumference; $\mathrm{DM}=$ diabetes mellitus; $\mathrm{Cr}=$ creatinine; $\mathrm{K}=$ potassium; $\mathrm{UA}=$ uric acid; $\mathrm{TG}=$ triglyceride; $\mathrm{HDL}-\mathrm{C}$ = high-density lipoprotein cholesterol; $\mathrm{GA}=$ glycoalbumin.

\section{Results}

Table 1 shows that no significant differences in baseline patient characteristics were observed between the group treated with CCB-based therapy and the group treated with telmisartan-based therapy. At the end of the study, the numbers of patients in the group treated with CCB-based therapy who had been prescribed additional antihypertensive medication were as follows: 5 for losartan, 4 for valsartan, 2 for olmesartan, 7 for candesartan, 2 for enalapril, 3 for diuretics, and 10 for $\beta$-blockers. In the group treated with telmisartan-based therapy, 3 patients were additionally treated with diuretics and 17 were treated with CCB.

Table 2 shows the changes in BP during the 12 -month treatment period. No significant differences in the baseline BP were observed between the group treated with CCB-based therapy and the group treated with telmisartan-based therapy. The clinical systolic BP decreased significantly after the CCB-based therapy and also de- 
Table 2. Changes in blood pressure during the study period

\begin{tabular}{lcccccc}
\hline \multirow{2}{*}{ Therapy } & \multicolumn{2}{c}{ CCB-based } & & \multicolumn{2}{c}{ Telmisartan-based } & \multirow{2}{*}{$\begin{array}{c}\text { p (between } \\
\text { therapies) }\end{array}$} \\
\cline { 2 - 3 } & baseline & 12 months & & baseline & 12 months & \\
\hline Clinical SBP & $155 \pm 19$ & $134 \pm 12^{*}$ & & $156 \pm 14$ & $136 \pm 13^{*}$ & 0.935 \\
Clinical DBP & $95 \pm 11$ & $85 \pm 10^{*}$ & & $101 \pm 10$ & $87 \pm 11^{*}$ & 0.232 \\
24-hour SBP & $138 \pm 18$ & $130 \pm 13$ & & $144 \pm 11$ & $128 \pm 11^{*}$ & 0.053 \\
24-hour DBP & $88 \pm 9$ & $80 \pm 8^{*}$ & & $91 \pm 9$ & $81 \pm 7^{*}$ & 0.045 \\
Daytime SBP & $142 \pm 20$ & $135 \pm 14$ & & $148 \pm 10$ & $132 \pm 11^{*}$ & 0.045 \\
Daytime DBP & $88 \pm 12$ & $83 \pm 9^{*}$ & & $94 \pm 7$ & $84 \pm 8^{*}$ & 0.050 \\
Nighttime SBP & $125 \pm 20$ & $116 \pm 12$ & & $131 \pm 17$ & $113 \pm 13^{*}$ & 0.124 \\
Nighttime DBP & $76 \pm 11$ & $71 \pm 9$ & & $83 \pm 11$ & $72 \pm 9^{*}$ & 0.076 \\
Nocturnal decrease & $18 \pm 16$ & $19 \pm 12$ & & $17 \pm 13$ & $18 \pm 12$ & 0.994 \\
Morning surge & $44 \pm 12$ & $47 \pm 20$ & & $47 \pm 20$ & $43 \pm 17$ & 0.317 \\
SBP variability & $20 \pm 4$ & $20 \pm 5$ & & $19 \pm 5$ & $19 \pm 5$ & 0.763 \\
\hline
\end{tabular}

Units are $\mathrm{mm} \mathrm{Hg}$. Data are the means \pm SD. SBP $=$ Systolic blood pressure; $\mathrm{DBP}=$ diastolic blood pressure. ${ }^{*} \mathrm{p}<0.05$ vs. the baseline value.

creased significantly after the telmisartan-based therapy, although no significant difference was observed between the two groups. The clinical diastolic BP decreased significantly after the CCB-based therapy, and also declined significantly after the telmisartan-based therapy, although no significant difference was observed between the two groups. The 24-hour ambulatory systolic BP decreased significantly after the telmisartan-based therapy, whereas it did not change significantly after the CCBbased therapy. The 24-hour ambulatory diastolic BP decreased significantly after both the CCB-based therapy and the telmisartan-based therapy. The reduction in the 24-hour ambulatory diastolic BP after the telmisartanbased therapy was significantly greater than that after the CCB-based therapy. The daytime systolic BP decreased significantly after the telmisartan-based therapy, whereas it did not change significantly after the CCB-based therapy. The reduction in the daytime systolic BP after the telmisartan-based therapy was significantly greater than that after the CCB-based therapy. The daytime diastolic BP decreased significantly after the CCB-based therapy and also decreased significantly after the telmisartan-based therapy, although no significant difference was observed between the two groups. The nighttime systolic BP decreased significantly after the telmisartanbased therapy, but did not change significantly after the CCB-based therapy. The nighttime diastolic BP decreased significantly after the telmisartan-based therapy, but did not change significantly after the CCB-based therapy. The reduction in the nighttime diastolic pres- sure after the telmisartan-based therapy was significantly greater than that after the CCB-based therapy. No significant changes in the nocturnal decrease in systolic BP, the morning systolic BP surge or the systolic BP variability were seen during the 12-month observation period in either group.

Figure 1 shows the change in the BNP, UAE and arterial stiffness as assessed by CAVI, AI and MAX-IMT in both groups. The log UAE level decreased significantly from $1.03 \pm 0.66$ to $0.57 \pm 0.52$ after the telmisartanbased therapy, whereas it did not change significantly after the CCB-based therapy. CAVI decreased significantly from $8.2 \pm 1.0$ to $7.7 \pm 0.9$ after the telmisartan-based therapy, whereas it did not change significantly after the CCB-based therapy. CAVI also decreased significantly from 100 to $93.7 \pm 9.8 \%$ after the telmisartan-based therapy when the baseline values of CAVI were adjusted, but did not change significantly after the CCB-based therapy. No significant changes in the levels of BNP, AI or MAXIMT were observed in either group.

Figure 2 shows the changes in the metabolic parameters in both groups. During the 12-month observation period, the serum LDL-C level decreased significantly from $130 \pm 34$ to $119 \pm 32 \mathrm{mg} / \mathrm{dl}$ after the CCB-based treatment and also decreased significantly from $121 \pm 24$ to $115 \pm 29 \mathrm{mg} / \mathrm{dl}$ after the telmisartan-based therapy. However, no significant difference was observed between the two groups. The plasma ARC increased significantly from $9.6 \pm 8.7$ to $46.7 \pm 84.7 \mathrm{pg} / \mathrm{ml}$ after the telmisartanbased therapy, whereas no significant change was seen af- 

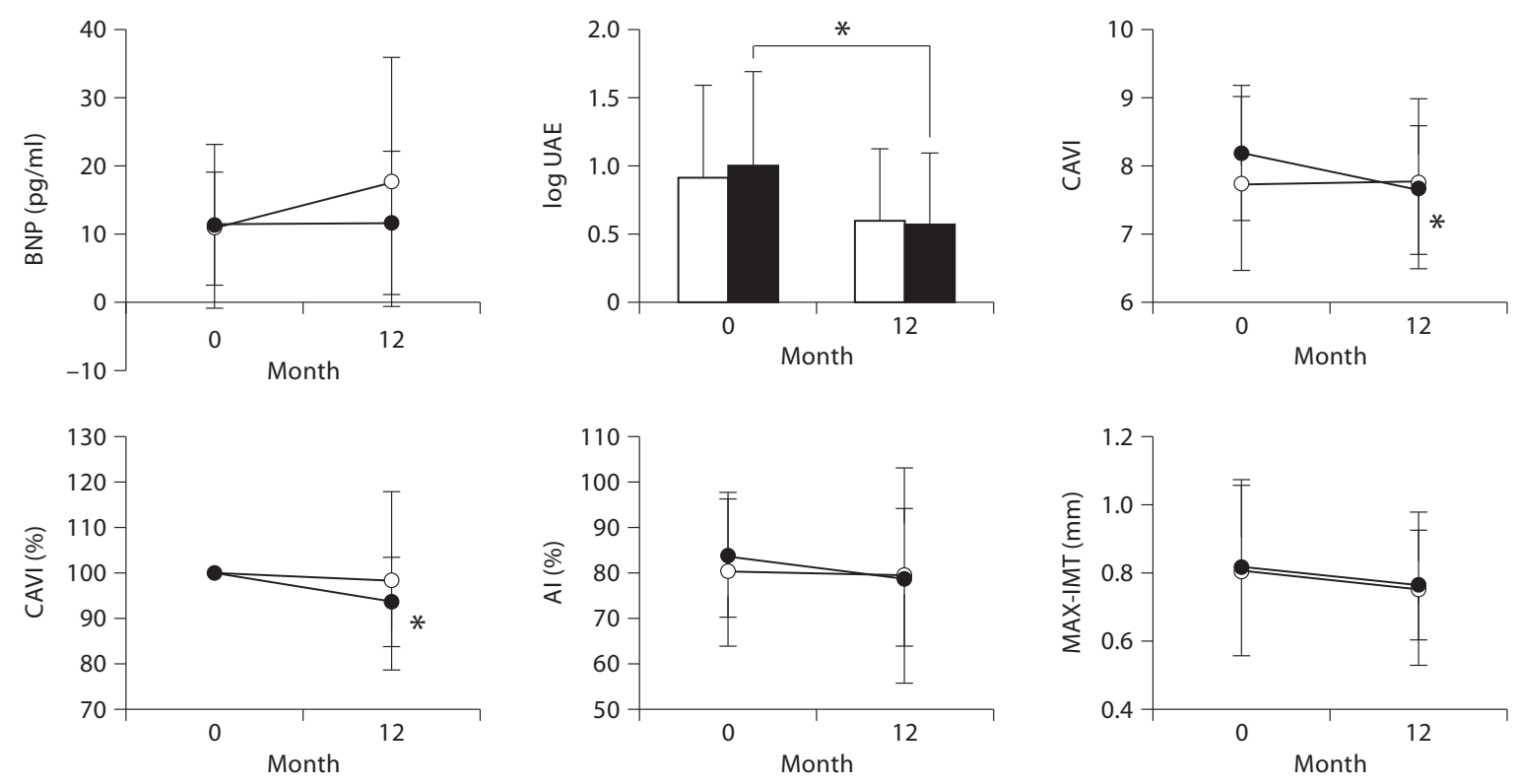

Fig. 1. Serum BNP, log UAE, AI, CAVI, the baseline adjusted CAVI and MAX-IMT at baseline and after 12 months of treatment in the CCB-based therapy (open circles) and the telmisartan-based therapy (closed circles) groups. ${ }^{*} \mathrm{p}<0.05$ vs. the baseline value.

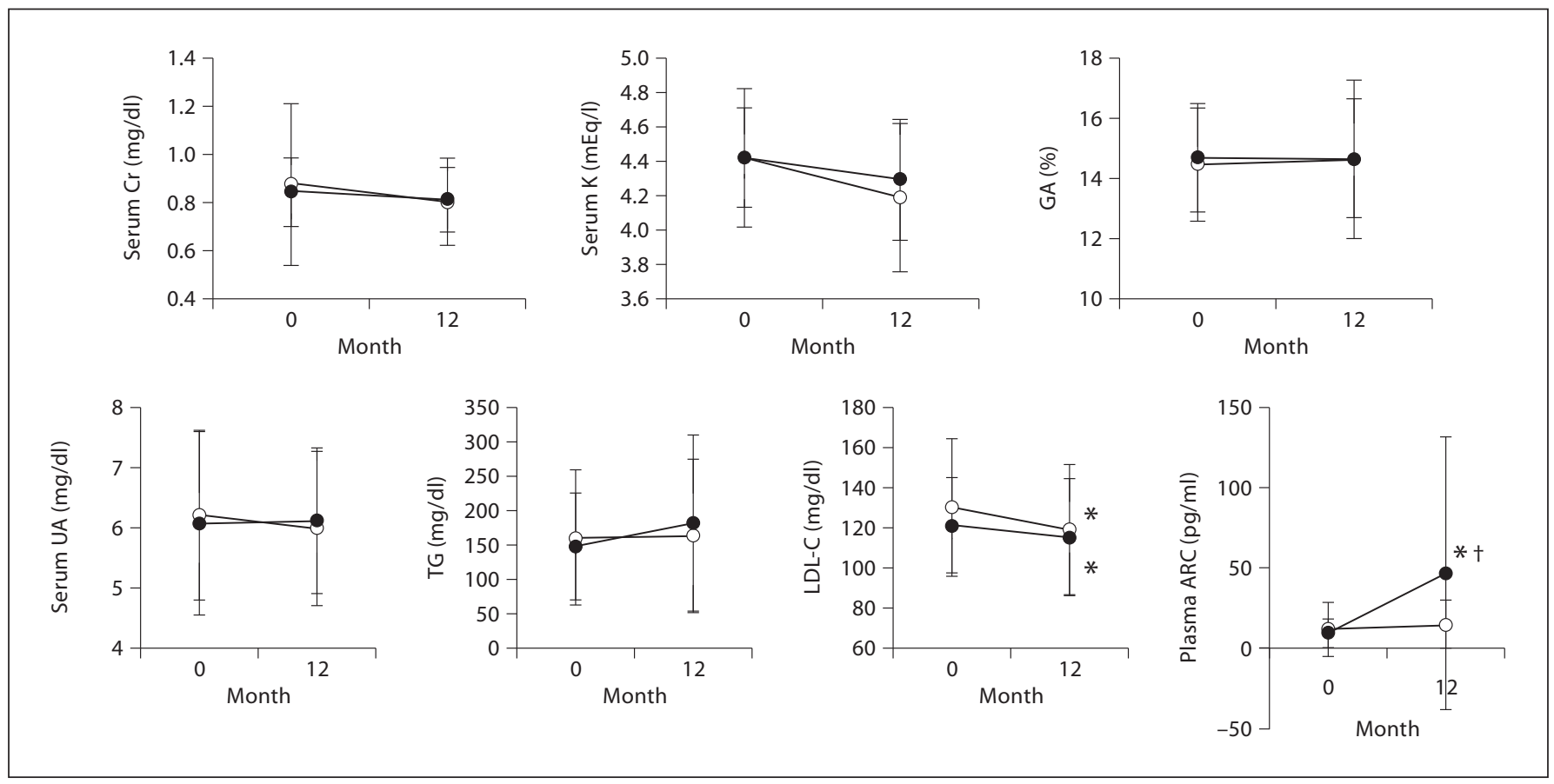

Fig. 2. Serum creatinine (Cr), serum $K$, glycoalbumin (GA), serum uric acid (UA), serum triglyceride (TG), serum LDL-C and plasma ARC at baseline and after 12 months of treatment in the CCB-based therapy (open circles) and the telmisartan-based therapy (closed circles) groups. ${ }^{*} \mathrm{p}<0.05$ vs. the baseline value; ${ }^{\dagger} \mathrm{p}<0.05 \mathrm{vs}$. the group treated with the CCB-based therapy. 
Table 3. Effects of percent changes in LDL, ARC, log UAE, clinical BP, 24-hour BP, daytime BP and nighttime BP on percent changes in CAVI after telmisartan-based therapy

\begin{tabular}{lrlrl}
\hline ANCOVA & Coefficient & SE & t test & $\mathrm{p}$ \\
\hline Intercept & -0.058 & 0.016 & -3.640 & 0.0008 \\
$\Delta$ LDL & 0.004 & 0.088 & 0.046 & 0.9638 \\
\hline Intercept & -0.044 & 0.019 & -2.281 & 0.0298 \\
$\Delta$ ARC & -0.005 & 0.002 & -2.382 & 0.0238 \\
\hline Intercept & -0.039 & 0.024 & -1.623 & 0.1151 \\
$\Delta$ log UAE & 0.064 & 0.051 & 1.263 & 0.2165 \\
\hline Intercept & -0.038 & 0.024 & -1.570 & 0.1241 \\
$\Delta$ clinical SBP & 0.182 & 0.157 & 1.154 & 0.2552 \\
\hline Intercept & -0.039 & 0.024 & -1.637 & 0.1093 \\
$\Delta$ clinical DBP & 0.167 & 0.142 & 1.181 & 0.2446 \\
\hline Intercept & -0.027 & 0.028 & -0.963 & 0.3427 \\
$\Delta$ 24-hour SBP & 0.290 & 0.204 & 1.423 & 0.1645 \\
\hline Intercept & -0.001 & 0.028 & -0.042 & 0.9671 \\
$\Delta$ 24-hour DBP & 0.449 & 0.199 & 2.515 & 0.0171 \\
\hline Intercept & -0.033 & 0.028 & -1.190 & 0.2429 \\
$\Delta$ daytime SBP & 0.246 & 0.205 & 1.201 & 0.2386 \\
\hline Intercept & -0.015 & 0.027 & -0.570 & 0.5729 \\
$\Delta$ daytime DBP & 0.397 & 0.193 & 2.054 & 0.0483 \\
\hline Intercept & -0.019 & 0.024 & -0.782 & 0.4398 \\
$\Delta$ nighttime SBP & 0.320 & 0.136 & 2.350 & 0.0251 \\
\hline Intercept & -0.024 & 0.021 & -1.114 & 0.2734 \\
$\Delta$ nighttime DBP & 0.305 & 0.120 & 2.531 & 0.0165 \\
\hline \multicolumn{1}{c}{ SB } & & & & \\
\hline
\end{tabular}

$\mathrm{SBP}=$ Systolic blood pressure; $\mathrm{DBP}=$ diastolic blood pressure.

ter the CCB-based therapy. The increase in the plasma ARC after the telmisartan-based therapy was significantly greater than that after the CCB-based therapy. No significant changes in the serum levels of creatinine, potassium, glycoalbumin, uric acid or triglyceride were seen during the 12-month observation period in either group.

In the present study, serum LDL-C, ARC, log UAE, clinical BP, 24-hour BP, daytime BP and nighttime BP changed significantly after the telmisartan-based therapy. As table 3 shows, we performed ANCOVA (analysis of covariance) to examine whether these variables affected the change in CAVI during telmisartan-based therapy. The changes in the plasma ARC, 24-hour diastolic BP, daytime diastolic BP and nighttime systolic and diastolic $\mathrm{BP}$ contributed significantly to the decrease in CAVI after the telmisartan-based therapy.

Effects of Telmisartan on Arterial Stiffness
Table 4. Effects of percent changes in LDL, ARC, CAVI, clinical $\mathrm{BP}, 24$-hour BP, daytime $\mathrm{BP}$ and nighttime $\mathrm{BP}$ on percent changes in log UAE after telmisartan-based therapy

\begin{tabular}{|c|c|c|c|c|}
\hline ANCOVA & Coefficient & SE & $\mathrm{t}$ test & $\mathrm{p}$ \\
\hline Intercept & -0.301 & 0.065 & -4.641 & $<0.0001$ \\
\hline$\Delta \mathrm{LDL}$ & 0.041 & 0.408 & 0.101 & 0.9201 \\
\hline Intercept & -0.265 & 0.085 & -3.130 & 0.0044 \\
\hline$\Delta \mathrm{ARC}$ & -0.013 & 0.009 & -1.438 & 0.1629 \\
\hline Intercept & -0.265 & 0.074 & -3.571 & 0.0012 \\
\hline$\Delta$ CAVI & 0.787 & 0.624 & 1.263 & 0.2165 \\
\hline Intercept & -0.271 & 0.113 & -2.403 & 0.0227 \\
\hline$\Delta$ clinical SBP & 0.265 & 0.773 & 0.342 & 0.7347 \\
\hline Intercept & -0.282 & 0.112 & -2.522 & 0.0172 \\
\hline$\Delta$ clinical DBP & 0.150 & 0.683 & 0.220 & 0.8276 \\
\hline Intercept & -0.222 & 0.121 & -1.830 & 0.0783 \\
\hline$\Delta 24$-hour SBP & 0.946 & 0.841 & 1.125 & 0.2705 \\
\hline Intercept & -0.224 & 0.127 & -1.761 & 0.0896 \\
\hline$\Delta 24$-hour DBP & 0.890 & 0.870 & 1.023 & 0.3152 \\
\hline Intercept & -0.230 & 0.128 & -1.793 & 0.0842 \\
\hline$\Delta$ daytime SBP & 0.858 & 0.898 & 0.956 & 0.3475 \\
\hline Intercept & -0.274 & 0.123 & -2.232 & 0.0341 \\
\hline$\Delta$ daytime DBP & 0.497 & 0.843 & 0.589 & 0.5606 \\
\hline Intercept & -0.215 & 0.094 & -2.287 & 0.0302 \\
\hline$\Delta$ nighttime SBP & 0.950 & 0.525 & 1.810 & 0.0815 \\
\hline Intercept & -0.262 & 0.093 & -2.812 & 0.0091 \\
\hline$\Delta$ nighttime DBP & 0.581 & 0.506 & 1.150 & 0.2602 \\
\hline
\end{tabular}

$\mathrm{SBP}=$ Systolic blood pressure $\mathrm{DBP}=$ diastolic blood pressure.

As table 4 shows, ANCOVA was also performed to examine whether the changes in the serum LDL-C, ARC, CAVI, clinical BP, 24-hour BP, daytime BP and nighttime $\mathrm{BP}$ affected the change in log UAE during the telmisartan-based therapy. However, none of the parameter changes contributed to the decrease in log UAE after the telmisartan-based therapy.

\section{Discussion}

This study demonstrated for the first time that telmisartan significantly improves CAVI, which reflects arterial stiffness in a manner that is less dependent on BP, compared with PWV in hypertensive patients [5]. The average coefficient of variation of CAVI is less than $5 \%$, 
which is small enough for clinical usage and indicates that CAVI has good reproducibility and validation of the measurement of arterial stiffness [13]. In addition, CAVI is a new marker for arterial stiffness since the previous study has reported that CAVI reflects the fibrosis of arterial walls histologically [5]. In the present study, telmisartan also significantly improved CAVI by $6.3 \%$ when the baseline values were adjusted, which was larger than the average coefficient of variation of the CAVI. The results of this study agree with those of previous studies, which showed improvements in vascular compliance after treatment with other ARBs [12]. In fact, previous studies have revealed that the long-term administration of telmisartan improved the PWV in spite of a BP-lowering efficacy comparable to those of other antihypertensive medications $[9,14]$. Since angiotensin II stiffens the vascular wall by constricting the vascular smooth muscle and promoting vascular wall remodeling, ARBs might improve arterial stiffness, at least in part, through BPindependent effects [7]. In the present study, ANCOVA showed that the changes in plasma ARC contributed significantly to the decrease in CAVI after the telmisartanbased therapy, although the changes in the 24-hour diastolic BP, daytime diastolic BP and nighttime systolic and diastolic $\mathrm{BP}$ also contributed significantly to the decrease in CAVI after the telmisartan-based therapy. Thus, the improvement of CAVI after telmisartan-based therapy might result from RAS blockade as well as antihypertensive effects. In addition, previous studies showed that telmisartan has the unique activity of enhancing PPAR$\gamma$, which plays critical roles in the vasculature [8]. In vascular endothelial cells, PPAR- $\gamma$ activation inhibits endothelial inflammation by suppressing inflammatory gene expression, leading to endothelial dysfunction [15]. In vascular smooth muscle cells, PPAR- $\gamma$ activation inhibits proliferation and migration, and promotes apoptosis [15]. Taken together, the properties of telmisartan, such as RAS blockade and PPAR- $\gamma$ activation, are thought to be involved in the improvement of CAVI. Since arterial stiffness is a powerful and independent risk factor for mortality in cardiovascular events [16], telmisartan could be a first-line antihypertensive drug with additional cardiovascular protective properties.

$\mathrm{AI}$ is another marker of arterial stiffness; this marker is associated with the plasma BNP because both markers reflect the central aortic pressure [13, 17]. Vascular stiffening causes an increase in the amplitude and early return of the reflected wave during systole, with augmentation of the central systolic BP and a resultant increase in AI [18]. Furthermore, the carotid IMT represents struc- tural changes like wall thickening and the formation of atherosclerotic plaques, which are correlated with a greater incidence of atherosclerosis in the coronary circulation and other large arteries [19]. Telmisartan has been shown to cause an insignificant reduction in AI [18] and significant decreases in both BNP [20] and IMT [21]. In the present study, telmisartan-based therapy also tended to decrease the AI and to regress the IMT, consistent with the above-mentioned studies, whereas telmisartan-based therapy did not decrease the BNP. While a previous study demonstrated a significant reduction in BNP after treatment with telmisartan when the initial baseline level was $27.8 \pm 21 \mathrm{pg} / \mathrm{ml}[20]$, the baseline level of BNP in the present study was $11.2 \pm 11.9 \mathrm{pg} / \mathrm{ml}$. Therefore, the plasma BNP level in the subjects in the present study might have been too low to detect any notable changes.

Albuminuria is an important predictor of cardiovascular events and of progression to end-stage renal disease in hypertensive patients $[22,23]$. In the present study, telmisartan-based therapy significantly reduced microalbuminuria in hypertensive patients. This result was consistent with those of previous studies showing that ARB treatment inhibits the progression to macroalbuminuria and overt proteinuria in diabetic patients with microalbuminuria $[24,25]$ and that telmisartan treatment reduces microalbuminuria in hypertensive patients [14]. The renoprotective effects exerted by telmisartan might be partly mediated by BP-independent mechanisms, since ANCOVA showed that the BP changes did not contribute to the decrease in log UAE after the telmisartan-based therapy. Telmisartan, a dual ARB/PPAR- $\gamma$ agonist, might have a great antiproteinuric effect [26] since treatment with telmisartan enabled a greater reduction in microalbuminuria than treatment with an ARB without any PPAR- $\gamma$ agonistic action [27]. The present study demonstrated a significant reduction in the log UAE value, even though ACE inhibitors or ARBs other than telmisartan were prescribed for $40 \%$ of the patients treated with CCB-based therapy. Thus, ARBs with properties that enhance PPAR- $\gamma$ might be useful for reducing microalbuminuria in hypertensive patients.

Telmisartan has been shown to be characterized by a balanced antihypertensive efficacy that fully covers a 24hour period, thereby antagonizing the adverse effects of early morning BP elevations on cardiovascular risk [19]. The beneficial effects of telmisartan on the 24-hour BP values consist not only of lowering the day time and nighttime $\mathrm{BP}$, but also effectively controlling BP variability $[28$, 29], which has been shown to be closely correlated with PWV [11], target-organ damage [30] and the incidence of
Kinouchi/Ichihara/Sakoda/ Kurauchi-Mito/Murohashi-Bokuda/Itoh 
cardiovascular events and mortality independent of the absolute BP load [31]. Previous studies have shown that the inhibitory effects of telmisartan on BP variability were associated with a reduction in sympathetic nerve activity in hypertensive patients with overt nephropathy [28]. Thus, telmisartan could be beneficial in hypertensive patients with a greater BP variability and/or autonomic dysfunction, such as elderly subjects and diabetic patients. Although reductions in morning surge and BP fluctuations were not observed in the present study, a sustained antihypertensive effect was observed throughout the 24-hour observation period among the subjects treated with telmisartan-based therapy.

ARB has also been shown to have a favorable lipid profile [32]. In the present study, telmisartan significantly decreased the serum level of LDL-C, which was in line with previous studies [33]. However, the reduction in the serum LDL-C levels after telmisartan-based therapy was similar to that observed after CCB-based therapy, probably because of the relatively large proportion of patients with prescriptions for ACE inhibitors or ARBs other than telmisartan among the patients treated with the CCB-based therapy. In the present study, the telmisartan-based therapy did not increase the serum levels of creatinine or potassium, which is consistent with the ONTARGET study (which demonstrated a similar number of patients whose serum creatinine levels and potassium levels increased after telmisartan or ramipril therapy) [34]. Thus, the present study also confirmed the metabolic safety of telmisartan.

In the present study, ARBs and CCBs were added in the group treated with CCBs and telmisartan, respectively, unless the target BP was achieved. The Candesartan Antihypertensive Survival Evaluation in Japan (CASE-J) trial revealed that $\mathrm{BP}$ reduction is important for the cardiovascular protection at an early stage, while the contri- bution of RAS inhibition to the reduction of the cardiovascular events is apparent in a delayed fashion [35]. Based on the CASE-J trial, one would expect that the early initiation of ARBs will be beneficial for the inhibition of the arterial stiffness. In fact, the current study clearly provided evidence that the arterial stiffness assessed by CAVI significantly improved in the group treated with telmisartan from the start of the study, whereas the improvement of the arterial stiffness in the CCB-based therapy was not as much as that in the telmisartan-based therapy, even after the addition of ARB. Therefore, telmisartan should be the first drug to be initiated in view of the protection from the arterial stiffness.

Some limitations in the interpretation of the results of the present study exist. First, the trial population was comparatively small and the observation period was relatively short. A longer observation with a larger number of subjects might elucidate the beneficial and adverse effects of telmisartan more clearly. In addition, prognostic events were not examined in the present study. Further studies are needed to confirm the benefits and safety of telmisartan-based therapy.

In conclusion, telmisartan-based therapy exerted beneficial effects on arterial stiffness, as assessed using CAVI, albuminuria, 24-hour BP and metabolism compared with CCB-based therapy. Since these markers are known to influence the future risk for cardiovascular events in hypertensive patients, telmisartan could well be a firstline antihypertensive drug for the treatment of hypertensive patients.

\section{Acknowledgment}

We appreciate the skillful secretarial work of Ms. Chika Miki.

\section{References}

1 Laurent S, Boutouyrie P, Asmar R, Gautier I, Laloux B, Guize L, Ducimetiere P, Benetos A: Aortic stiffness is an independent predictor of all-cause and cardiovascular mortality in hypertensive patients. Hypertension 2001; 37:1236-1241.

2 Mitchell G: Effects of central arterial aging on the structure and function of the peripheral vasculature: implications for end-organ damage. J Appl Physiol 2008;105:1652-1660.

3 Safar M: Systolic blood pressure, pulse pressure and arterial stiffness as cardiovascular risk factors. Curr Opin Nephrol Hypertens 2001;10:257-261.

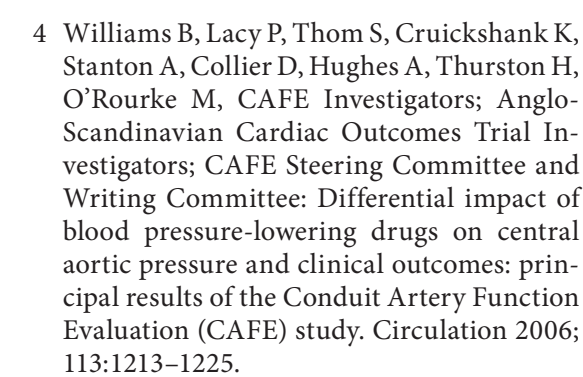

5 Ichihara A, Yamashita N, Takemitsu T, Kaneshiro Y, Sakoda M, Kurauchi-Mito A, Itoh $\mathrm{H}$ : Cardio-ankle vascular index and ankle pulse wave velocity as a marker of arterial fibrosis in kidney failure treated by hemodialysis. Am J Kidney Dis 2008;57: 947-955.

-6 Okura T, Watanabe S, Kurata M, Manabe S, Koresawa M, Irita J, Enomoto D, Miyoshi K, Fukuoka T, Higaki J: Relationship between cardio-ankle vascular index (CAVI) and carotid atherosclerosis in patients with essential hypertension. Hypertension Res 2007; 30:335-340. 
7 Mahmud A, Feely J: Effect of angiotensin II receptor blockade on arterial stiffness: beyond blood pressure reduction. Am J Hypertens 2002;15:1092-1095.

-8 Benson S, Pershadsingh H, Ho C, Chittiboyina A, Desai P, Pravenec M, Qi N, Wang J, Avery M, Kurtz T: Identification of telmisar$\tan$ as a unique angiotensin II receptor antagonist with selective PPAR $\gamma$-modulating activity. Hypertension 2004;43:993-1002.

$>9$ Uchida H, Nakamura Y, Kaihara M, Sugimoto T, Norii H, Sasaki M, Sato H, Makino H: Practical efficacy of telmisartan for decreasing morning home blood pressure and pulse wave velocity in patients with mild-to-moderate hypertension. Hypertension Res 2004; 27:545-550.

10 Pontremoli R, Leoncini G, Viazzi F, Ratto E, Vaccaro V, Falqui V, Parodi A, Conti N, Tomolillo C, Deferrari G: Evaluation of subclinical organ damage for risk assessment and treatment in the hypertensive patient: role of microalbuminuria. J Am Soc Nephrol 2006;17(4 Suppl 2):S112-S114.

-11 Ichihara A, Kaneshiro Y, Takemitsu T, Sakoda M: Effects of amlodipine and valsartan on vascular damage and ambulatory blood pressure in untreated hypertensive patients. J Hum Hypertens 2006;10:787-794.

-12 Kinouchi K, Ichihara A, Sakoda M, Kurauchi-Mito A, Itoh H: Safety and benefits of a tablet combining losartan and hydrochlorothiazide in Japanese diabetic patients with hypertension. Hypertension Res 2009;32: 1143-1147.

13 Sakuragi S, Maruo T, Taniguchi M, Nagase S, Nakamura K, Kusano K, Ohe T: Radial augmentation index associated with increase in b-type natriuretic peptide in patients with hypertension. Int J Cardiol 2008;130:414419.

14 Morimoto S, Yano Y, Maki K, Sawada K: Renal and vascular protective effects of telmisartan in patients with essential hypertension. Hypertension Res 2006;29:567-572.

-15 Duan S, Usher M, Mortensen R: Peroxisome proliferator-activated receptor-mediated effects in the vasculature. Circ Res 2008;102: 283-294.

16 Willum-Hansen T, Staessen J, Torp-Pedersen C, Rasmussen S, Thijs L, Ibsen H, Jeppesen J: Prognostic value of aortic pulse wave velocity as index of arterial stiffness in the general population. Circulation 2006;113: 664-670.

17 Dawson A, Davies J, Morris A, Struthers A: B-type natriuretic peptide is associated with both augmentation index and left ventricular mass in diabetic patients without heart failure. Am J Hypertens 2005; 18:1586-1591.
18 Asmar R, Gosse P, Topouchian J, N'tela G, Dudley A, Shepherd G: Effects of telmisartan on arterial stiffness in type 2 diabetes patients with essential hypertension. J Renin Angiotensin Aldosterone Syst 2002;3:176180.

19 Grassi G, Quarti-Trevano F, Mancia G: Review: cardioprotective effects of telmisartan in uncomplicated and complicated hypertension. J Renin Angiotensin Aldosterone Syst 2008;9:66-74.

20 Symeonides P, Koulouris S, Vratsista E, Triantafyllou $\mathrm{K}$, Ioannidis $\mathrm{G}$, Thalassinos $\mathrm{N}$, Katritsis D: Both ramipril and telmisartan reverse indices of early diabetic cardiomyopathy: a comparative study. Eur J Echocardiogr 2006;8:480-486.

21 Petrovic I, Petrovic D, Vukovic N, Zivanovic B, Dragicevic J, Vasiljevic Z, Babic R: Ventricular and vascular remodelling effects of the angiotensin II receptor blocker telmisartan and/or the angiotensin-converting enzyme inhibitor ramipril in hypertensive patients. J Int Med Res 2005;33:39A-49A.

22 Garg J, Bakris G: Microalbuminuria: marker of vascular dysfunction, risk factor for cardiovascular disease. Vasc Med 2002;7:35-43.

23 Gerstein H, Mann J, Yi Q, Zinman B, Dinneen S, Hoogwerf B, Halle J, Young J, Rashkow A, Joyce C, Nawaz S, Yusuf S: Albuminuria and risk of cardiovascular events, death, and heart failure in diabetic and nondiabetic individuals. JAMA 2001;286:421-426.

24 Parving H, Lehnert H, Brochner-Mortensen J, Gomis R, Andersen S, Arner P; Irbesartan in Patients with Type 2 Diabetes and Microalbuminuria Study Group: The effect of irbesartan on the development of diabetic nephropathy in patients with type 2 diabetes. $\mathrm{N}$ Engl J Med 2001;345:870-878.

25 Viberti G, Wheeldon N; MicroAlbuminuria Reduction With VALsartan (MARVAL) Study Investigators: Microalbuminuria reduction with valsartan in patients with type 2 diabetes mellitus: a blood pressure-independent effect. Circulation 2002;106:672678.

26 Bichu P, Nistala R, Khan A, Sowers J, Whaley-Connell A: Angiotensin receptor blockers for the reduction of proteinuria in diabetic patients with overt nephropathy: results from the AMADEO study. Vasc Health Risk Manag 2009;5:129-140.
27 Yano Y, Hoshide S, Ishikawa J, Noguchi C, Tukui D, Takanori H, Tada M, Kanemaru Y, Yano A, Ishikawa S, Shimada K, Kario K: The differential effects of angiotensin II type 1 receptor blockers on microalbuminuria in relation to low-grade inflammation in metabolic hypertensive patients. Am J Hypertens 2007;20:565-572.

28 Masuda S, Tamura K, Wakui H, Kanaoka T, Ohsawa M, Maeda A, Dejima T, Yanagi M, Azuma K, Umemura S: Effects of angiotensin II type 1 receptor blocker on ambulatory blood pressure variability in hypertensive patients with overt diabetic nephropathy. Hypertension Res 2009;32:950-955.

-29 Nakamoto H, Nishida E, Ryuzaki M, Sone M, Suzuki H, Yoshimoto M, Itagaki K: Effect of telmisartan and amlodipine on home blood pressure by monitoring newly developed telemedicine system: monitoring test by using telemedicine. Telmisartan's effect on home blood pressure (TelTelbosu). Clin Exp Hypertens 2008;30:57-67.

>30 Mancia G, Grassi G: Mechanisms and clinical implications of blood pressure variablity. J Cardiovasc Pharmacol 2000;35:S15-S19.

-31 Mancia G, Bombelli M, Facchetti R, et al: Long-term prognostic value of blood pressure variability in the general population: results of the Pressioni Arteriose Monitorate e Loro Associazioni Study. Hypertension 2007;49:1265-1270.

-32 Kyvelou S, Vyssoulis G, Karpanou E, Adamopoulos D, Zervoudaki A, Pietri P, Stefanadis C: Effects of antihypertensive treatment with angiotensin II receptor blockers on lipid profile: an open multi-drug comparison trial. Hellenic J Cardiol 2006;47:21-28.

-33 Derosa G, Ragonesi P, Mugellini A, Ciccarelli L, Fogari R: Effects of telmisartan compared with eprosartan on blood pressure control, glucose metabolism and lipid profile in hypertensive, type 2 diabetic patients: a randomized, double-blind, placebo-controlled 12-month study. Hypertension Res 2004;27:457-464.

34 ONTARGET Investigators; Yusuf S, Teo KK, Pogue J, Dyal L, Copland I, Schumacher H, Dagenais G, Sleight P, Anderson C: Telmisartan, ramipril, or both in patients at high risk for vascular events. N Engl J Med 2008; 358:1547-1559.

-35 Ogihara T, Nakao K, Fukui T, Fukiyama K, Ueshima K, Oba K, Sato T, Saruta T: Effects of candesartan compared with amlodipine in hypertensive patients with high cardiovascular risks: candesartan antihypertensive survival evaluation in Japan trial. Hypertension 2008;51:393-398. 